\title{
Dynamic unmagnetized plasma in the diamagnetic cavity around comet 67P/Churyumov-Gerasimenko
}

\author{
Rajkumar Hajra, ${ }^{1 \star}$ Pierre Henri, ${ }^{1}$ Xavier Vallières, ${ }^{1}$ Jerome Moré,${ }^{1}$ Nicolas Gilet, ${ }^{1}$ \\ Gaetan Wattieaux, ${ }^{2}$ Charlotte Goetz, ${ }^{3}$ Ingo Richter, ${ }^{3}$ Bruce T. Tsurutani, ${ }^{4}$ \\ Herbert Gunell, ${ }^{5,6}$ Hans Nilsson, ${ }^{7}$ Anders I. Eriksson, ${ }^{8}$ Zoltan Nemeth, ${ }^{9}$ \\ James L. Burch ${ }^{10}$ and Martin Rubin ${ }^{11}$ \\ ${ }^{1}$ Laboratoire de Physique et Chimie de l'Environnement et de l'Espace (LPC2E), CNRS, F-45071 Orléans, France \\ ${ }^{2}$ LAPLACE, Université de Toulouse, CNRS, F-31062 Toulouse, France \\ ${ }^{3}$ Institut für Geophysik und extraterrestrische Physik, TU Braunschweig, Mendelssohnstr. 3, D-38106 Braunschweig, Germany \\ ${ }^{4}$ Jet Propulsion Laboratory, California Institute of Technology, 4800 Oak Grove Drive, Pasadena, CA 91109, USA \\ ${ }^{5}$ Royal Belgian Institute for Space Aeronomy (BIRA-IASB), Avenue Circulaire 3, B-1180 Brussels, Belgium \\ ${ }^{6}$ Department of Physics, UmeåUniversity, SE-901 87 Umeå, Sweden \\ ${ }^{7}$ Swedish Institute of Space Physics, PO Box 812, SE-981 28 Kiruna, Sweden \\ ${ }^{8}$ Institutet för rymdfysik, Angstrom Laboratory, Lagerhyddsvagen 1, SE-75120 Uppsala, Sweden \\ ${ }^{9}$ Wigner Research Centre for Physics, Konkoly-Thege M. Rd. 29-33 Budapest, Hungary \\ ${ }^{10}$ Southwest Research Institute, PO Drawer 28510, San Antonio, TX 78228-0510, USA \\ ${ }^{11}$ Physikalisches Institut, Universität Bern, Sidlerstr. 5, CH-3012 Bern, Switzerland
}

Accepted 2018 January 4. Received 2017 December 18; in original form 2017 September 27

\begin{abstract}
The Rosetta orbiter witnessed several hundred diamagnetic cavity crossings (unmagnetized regions) around comet 67P/Churyumov-Gerasimenko during its two year survey of the comet. The characteristics of the plasma environment inside these diamagnetic regions are studied using in situ measurements by the Rosetta Plasma Consortium instruments. Although the unmagnetized plasma density has been observed to exhibit little dynamics compared to the very dynamical magnetized cometary plasma, we detected several localized dynamic plasma structures inside those diamagnetic regions. These plasma structures are not related to the direct ionization of local cometary neutrals. The structures are found to be steepened, asymmetric plasma enhancements with typical rising-to-descending slope ratio of $\sim 2.8( \pm 1.9)$, skewness $\sim 0.43( \pm 0.36)$, mean duration of $\sim 2.7( \pm 0.9)$ min and relative density variation $\Delta N / N$ of $\sim 0.5( \pm 0.2)$, observed close to the electron exobase. Similar steepened plasma density enhancements were detected at the magnetized boundaries of the diamagnetic cavity as well as outside the diamagnetic region. The plausible scalelength and propagation direction of the structures are estimated from simple plasma dynamics considerations. It is suggested that they are large-scale unmagnetized plasma enhancements, transmitted from the very dynamical outer magnetized region to the inner magnetic field-free cavity region.
\end{abstract}

Key words: methods: data analysis - methods: observational-comets: general-comets: individual: 67P/Churyumov-Gerasimenko.

\section{INTRODUCTION}

The diamagnetic cavity (magnetic field-free region) near the cometary nucleus is one of the most interesting features of the plasma environment of active comets. The cometary plasma from the active outgassing comet exerts pressure on the incoming solar

^E-mail: rhajra@cnrs-orleans.fr wind. This mechanism leads to the formation of a near-nucleus solar wind cavity void of any solar wind ions (e.g. Behar et al. 2017; Nilsson et al. 2017) and a diamagnetic cavity free from interplanetary magnetic fields (e.g. Neubauer et al. 1986; Cravens 1987; Ip \& Axford 1987). In this work, we concentrate on the later. A diamagnetic cavity was first detected around the comet 1P/Halley at a heliocentric distance of $\sim 0.9$ au from the Sun by the Giotto spacecraft in 1986 (Neubauer et al. 1986). During a $\sim 2$ min period of the Halley fly-by with a closest approach distance of $\sim 500 \mathrm{~km}$, 
the cavity was estimated to expand to $>4000 \mathrm{~km}$ from the comet nucleus. Recently, Goetz et al. (2016a) identified a total of 665 diamagnetic cavity crossings during a $\sim 2$ yr-long in situ monitoring of the comet 67P/Churyumov-Gerasimenko (hereafter referred to as 67P; Churyumov \& Gerasimenko 1972) by the Rosetta spacecraft (Glassmeier et al. 2007a). The cavity crossings were detected between 2015 April and 2016 February at heliocentric distances ranging from $\sim 1.25$ to $2.4 \mathrm{au}$. They were observed at cometocentric distances varying between $\sim 40$ and $380 \mathrm{~km}$. When taking into account the variation of cometary outgassing activity, Henri et al. (2017) have shown that most cavity crossings were observed close to the comet electron exobase, representing the transition region between collisional to collisionless electrons. Nemeth et al. (2016) and Madanian et al. (2017) reported short-term dropouts of suprathermal (150-200 eV) electron fluxes accompanied by longer duration $100 \mathrm{eV}$ electron flux attenuations around the regions. Although the magnetized plasma exhibits steep dynamic/compressible structures outside the diamagnetic cavity, both the plasma and the cometary neutrals inside the diamagnetic cavity are found to be rather constant, with an unmagnetized plasma density that scales with the distance to the comet nucleus (Henri et al. 2017).

However, we observed that inside $\sim 15$ percent of the longduration ( $\geq 5 \mathrm{~min}$ ) diamagnetic cavity crossings when plasma measurements were available (Henri et al. 2017), the unmagnetized cometary plasma exhibits significant density variability with respect to the steady neutral background. In this paper, we analyse the diamagnetic cavity crossings, where such significant plasma density enhancements are observed. The aim of this work is to understand and characterize the dynamics of the unmagnetized cometary plasma inside the diamagnetic region(s) surrounding comet 67P.

\section{DATA ANALYSES AND RESULTS}

The diamagnetic cavity crossings are identified by the three-axial fluxgate MAGnetometers of the Rosetta Plasma Consortium (RPCMAG; Glassmeier et al. 2007b). A detailed description of the magnetic field-free region detection around $67 \mathrm{P}$ can be found in Goetz et al. (2016a,b). Measurements from the mutual impedance probe (RPC-MIP; Trotignon et al. 2007) are used to characterize the plasma density around comet 67P (e.g. Hajra et al. 2017). The cometary neutral density is obtained from the COmet Pressure Sensor in the Rosetta Orbiter Spectrometer for Ion and Neutral Analysis (ROSINA/COPS; Balsiger et al. (2007)).

In the following subsections, we first present two case studies of unmagnetized cometary plasma enhancements observed during diamagnetic cavity crossings encountered on 2015 July 30. They are followed by a statistical study of the 23 unmagnetized plasma enhancements observations.

\subsection{Diamagnetic cavity crossing around 09 UT on 2015 July 30}

Fig. 1 shows observations of the plasma environment around 67P, at the spacecraft position, during a diamagnetic cavity crossing detected on 2015 July 30. The Rosetta spacecraft was at a cometocentric distance $R$ of $\sim 179 \mathrm{~km}$ from $67 \mathrm{P}$ and at a heliocentric distance of $\sim 1.3$ au. The amplitude of the magnetic field $B_{o} \lesssim 1$ nT, from 08:53:48 UT to 09:13:42 UT, indicates that the diamagnetic cavity crossing lasted $\sim 20 \mathrm{~min}$ (Fig. 1d). The magnetized regions around the diamagnetic cavity are shown by shadings. The magnetic field variations are consistent with the observations reported in Goetz et al. (2016a,b). In particular, the outbound crossing is

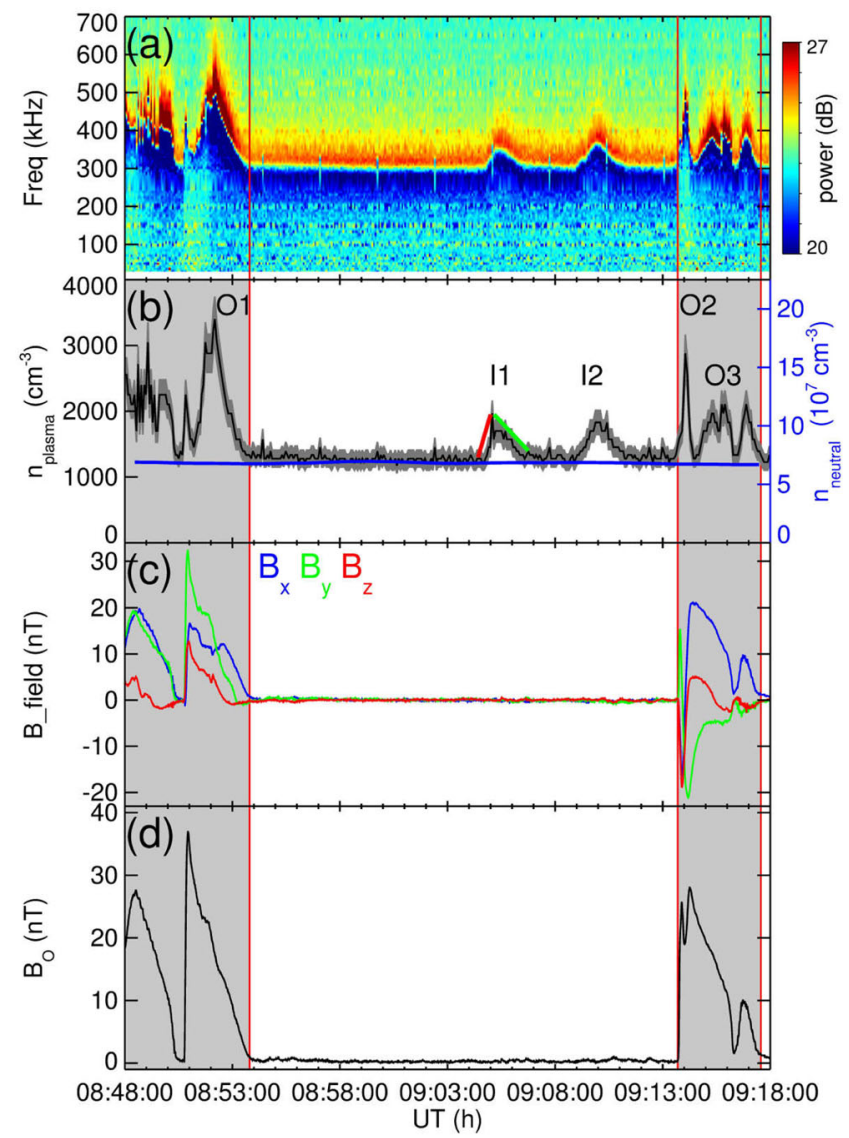

Figure 1. Observations of the cometary plasma environment around a diamagnetic cavity crossing at $\sim 09$ UT on 2015 July 30 . (a) The mutual impedance spectrogram. The colour bar at the right-hand side shows the power in $\mathrm{dB}$, using voltage reference level of $0.6 \mu \mathrm{V} \mathrm{Hz}^{-1 / 2}$, taking into account the signal received in vacuum. (b) The estimated electron density (black line) and neutral gas density (blue line). The red and green lines show the rising and descending slopes, respectively, of the enhanced plasma structure I1. The grey area along $n_{\text {plasma }}$ shows the uncertainty in measurement. (c) The magnetic field components (in CSEQ coordinate system) $B_{x}$ (blue), $B_{y}$ (green), $B_{z}$ (red), and (d) magnitude $B_{o}$, respectively. The magnetized regions are shaded.

sharper than the inbound crossing. The average magnetic field amplitudes before and after the cavity crossings are $\sim 16$ and $\sim 13 \mathrm{nT}$, respectively. Large fluctuations are observed in the magnetic field components (Fig. 1c). Another notable feature in this example is the positive-to-negative reversal of $B_{y}$ before and after the cavity, respectively. The magnetic field is expressed in the cometocentric solar equatorial (CSEQ) coordinate system, where the $x$-axis points towards the Sun, the $z$-axis is the component of the solar north pole that is orthogonal to the $x$-axis, and the $y$-axis completes the right-handed coordinate system.

Fig. 1(a) shows the mutual impedance amplitude spectrogram, which exhibits a strong resonance close to the plasma frequency. It is used to estimate the plasma density, $n_{\text {plasma }}$, shown in Fig. 1(b). Plasma density variations below $\sim 10$ percent would not be detected because of the finite frequency resolution used in the RPCMIP operational mode used to retrieve the plasma density. This uncertainty is shown by grey area along $n_{\text {plasma }}$. Inside the diamagnetic cavity, the $n_{\text {plasma }}$ exhibits two significant, localized increases from 09:04:42 UT to 09:06:35 UT and from 09:08:53 UT to 09:11:19 UT, with durations of $\sim 113$ and $\sim 146 \mathrm{~s}$, respectively, above the 
Table 1. Plasma characteristics near the diamagnetic cavity crossing at $\sim 09$ ut on 2015 July 30.

\begin{tabular}{lcccc}
\hline & Duration $(\mathrm{s})$ & $\Delta N / N($ percent) & Rising-to-descending slope ratio & Skewness \\
\hline I1 (unmagnetized) & 113 & 60 & 4.2 & 0.44 \\
I2 (unmagnetized) & 146 & 49 & 1.1 & 0.23 \\
O1 & 161 & 130 & 1.6 & 0.53 \\
O2 & 38 & 125 & 2.7 & 0.68 \\
O3 & 78 & 72 & 2.0 & 0.56 \\
\hline
\end{tabular}

background plasma density of $\sim 1225 \mathrm{~cm}^{-3}$. These enhanced plasma structures inside the diamagnetic cavity are marked as I1 and I2, respectively (Fig. 1b). They are characterized by peak plasma densities of $\sim 1965$ and $1830 \mathrm{~cm}^{-3}$ recorded at 09:05:05 UT and 09:10:06 UT, respectively. The plasma increases are therefore $\sim 60$ per cent and $\sim 49$ per cent of the background $n_{\text {plasma }}$, respectively. Following the plasma density peak, the $n_{\text {plasma }}$ decreases with slower rates compared to the rates of the increases, implying asymmetric, steepened structures. The asymmetry of this structure is evaluated in two complementary ways: by its skewness and the ratio of the ascending-to-descending density profile. The skewness of structure I1 is $\sim 0.44$. Also, the structure I1 has a rising slope of $32.2 \mathrm{~cm}^{-3} \mathrm{~s}^{-1}$ and a smaller descending slope of $7.6 \mathrm{~cm}^{-3} \mathrm{~s}^{-1}$, that is, a rising-to-descending slope ratio of 4.2. An example of slope measurement is shown in Fig. 1(b). The rising slope is estimated from the plasma density change from the background plasma density before peak density to the peak density divided by the time interval between the two or the rising time-scale. This is shown by a red line in Fig. 1(b). Similarly, the descending slope is measured by the plasma density decrease from the peak to the background density after the peak divided by the corresponding descending time-scale. This is shown by a green line in Fig. 1(b). The rising-to-descending slope ratio may be taken as a proxy for asymmetry of the structure together with its skewness. The structure I 2 is less asymmetric with the rising and descending slopes of 8.3 and $7.5 \mathrm{~cm}^{-3} \mathrm{~s}^{-1}$, respectively, leading to a rising-to-descending slope ratio of 1.1 and skewness of $\sim 0.23$. It may be noted that during this interval there were no appreciable changes in the spacecraft trajectory and velocity.

For comparison, the blue curve in Fig. 1(b) shows the variation of cometary neutral density $n_{\text {neutral }}$. It is interesting to note that $n_{\text {neutral }}$ is almost constant around a value of $\sim 7 \times 10^{7} \mathrm{~cm}^{-3}$ during the entire interval shown. The cometary plasma has been shown to mainly originate from the photo-ionization and electron-impact ionization of the cometary neutrals (Galand et al. 2016; Heritier et al. 2017a). If the neutral density is constant, while the photoionization is expected to remain constant, a local increase in the energetic electrons and/or a change in neutral velocity can result in plasma density variations. At the time-scales of the observed unmagnetized plasma enhancements, both neutral velocity variations and/or suprathermal electron variations can hardly be estimated. However, Heritier et al. (2017b) have shown that the main source of ions near perihelion is photo-ionization, whereas the contribution of electron-impact ionization is much less significant at that time than in other periods of the mission. This is why we consider the electron-impact ionization to be negligible compared to the photo-ionization. Thus, the enhancement of the unmagnetized plasma (inside the diamagnetic cavity) should be mainly associated to some plasma dynamics process.

The unmagnetized plasma variation, inside the diamagnetic cavity, may be compared with the magnetized plasma, outside the cavity (shown by shaded regions). The diamagnetic cavity is ob- served to be bounded by a highly dynamic magnetized plasma. The $n_{\text {plasma }}$ varies between $\sim 1280$ and $\sim 3400 \mathrm{~cm}^{-3}$ before the cavity and between $\sim 1280$ and $\sim 2880 \mathrm{~cm}^{-3}$ after the cavity. The largeamplitude, compressible magnetized plasma variations are found to be well-correlated with the large-amplitude magnetic field variations at the edges of the cavity. Three magnetized enhanced plasma regions (outside the cavity) are marked as $\mathrm{O} 1, \mathrm{O} 2$, and $\mathrm{O} 3$ (Fig. 1b). The duration, amplitude, and asymmetry of the magnetized plasma enhancements $(\mathrm{O} 1, \mathrm{O} 2, \mathrm{O} 3)$ are compared with those of the unmagnetized plasma enhancements (I1, I2) in Table 1. Both the magnetized and unmagnetized structures are asymmetric, with larger rising slopes compared to the descending slopes, and the durations are comparable. However, it may be noted that unmagnetized plasma enhancements inside the cavity are smaller compared to the magnetized enhancements outside the contact surface of the cavity.

To take into account the variation of the outgassing cavity from one observation to another, and to enable direct comparisons with the undisturbed background unmagnetized plasma (Henri et al. 2017), the cometocentric distance $R$ is expressed in terms of the electron exobase or electron-neutral collisionopause $L_{\mathrm{ex}}: R^{*}=R / L_{\mathrm{ex}}$. The $L_{\mathrm{ex}}$ is defined as the boundary between an electron-neutral collisional region around the comet and a collisionless region far from the comet (Mandt et al. 2016). It is estimated as $L_{\mathrm{ex}}=\sigma_{\mathrm{en}} n_{\text {neutral }} R^{2}$, where $\sigma_{\text {en }}$ is the electron-neutral cross-section and $n_{\text {neutral }}$ is the neutral density measured at the spacecraft position (see Mandt et al. (2016) for more details). Although $\sigma_{\text {en }}$ depends on electron energy (Itikawa \& Mason 2005), we have taken $\sigma_{\text {en }}$ to be $\sim 5 \times 10^{-16} \mathrm{~cm}^{2}$, considering an averaged electron energy of $\sim 5 \mathrm{eV}$ corresponding to the warm electron population of freshly ionized electrons reported in Odelstad et al. (2015) and Eriksson et al. (2017) from LAngmuir Probe (RPC-LAP; Eriksson et al. 2007) measurements. During the observations reported in Fig. $1, R^{*} \sim 0.82$, implying that the spacecraft was located near the electron-neutral collisionopause.

\subsection{Diamagnetic cavity crossings around 06 UT on 2015 July 30}

Fig. 2 shows another example of diamagnetic cavity crossings detected on the same day (2015 July 30), when the Rosetta spacecraft was at a cometocentric distance of $\sim 179 \mathrm{~km}$ and at a heliocentric distance of $\sim 1.3 \mathrm{au}$, corresponding to a normalized cometocentric distance $R^{*}$ of $\sim 0.89$. Thus, these crossings were also located near the electron exobase. Three magnetic field-free regions are observed from 05:52:48 uT to 05:54:08 UT (duration $\sim 1.3 \mathrm{~min}$ ), from 05:56:14 UT to 06:07:50 UT ( $\sim 11.6 \mathrm{~min}$ ), and from 06:09:05 uT to 06:13:09 UT ( $\sim 4 \mathrm{~min})$, surrounded by magnetized regions with average $B_{o}$ fields of $\sim 11,8,8$, and $18 \mathrm{nT}$, respectively (Fig. 2d). The magnetized regions are shown by shadings. All boundary regions are characterized by large dynamic structures (Figs 2a, 2b). However, $n_{\text {plasma }}$ is almost constant, with a value of $\sim 1100 \mathrm{~cm}^{-3}$, during two short-duration cavity crossings, whereas a large dynamic plasma structure is detected inside the second cavity crossing (05:56:14 UT 


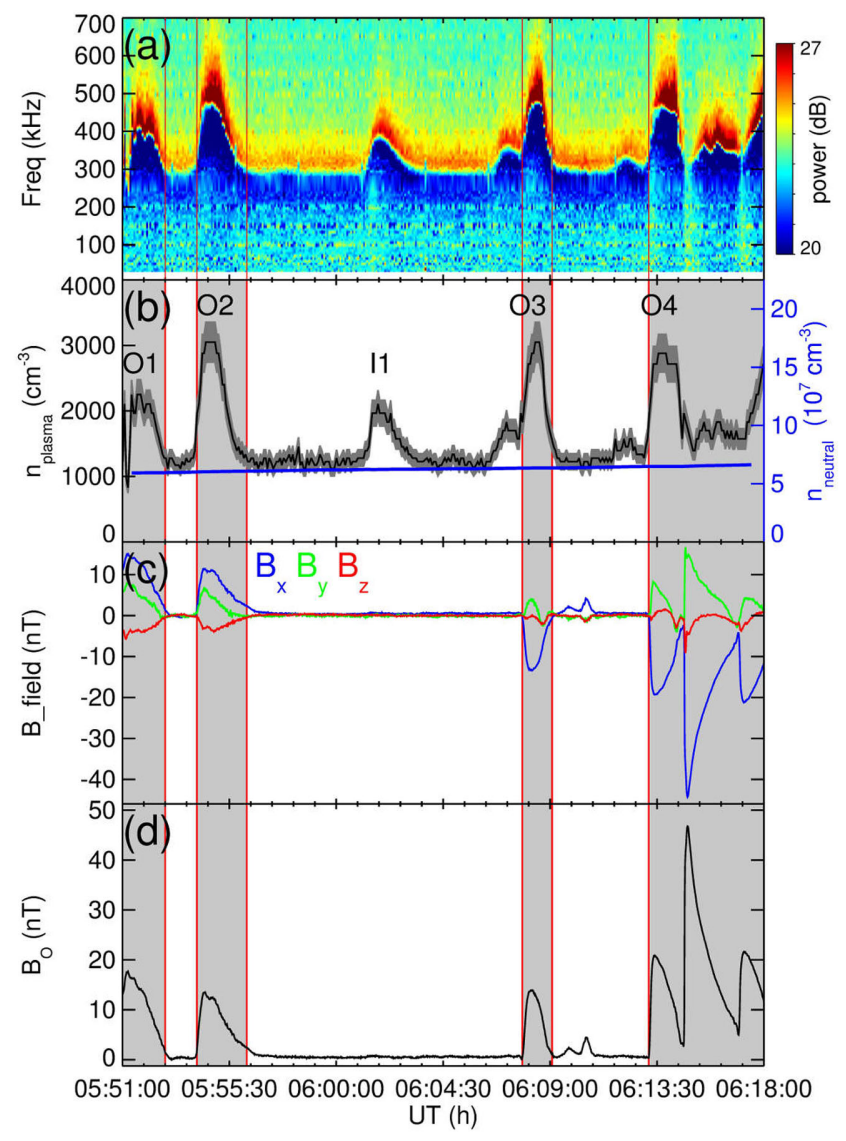

Figure 2. Observations of the cometary plasma environment around the diamagnetic cavity crossings at $\sim 06$ UT on 2015 July 30 . The panels are in the same format as in Fig. 1.

to $06: 07: 50$ ut) (Fig. 2b). It may be noted that the cometary neutral density is almost constant with $n_{\text {neutral }} \sim 6 \times 10^{7} \mathrm{~cm}^{-3}$ during the entire period shown in Fig. 2. This again indicates that plasma dynamical processes are involved in the unmagnetized plasma density increases rather than local ionization (photo- or electron-impact) of the cometary neutrals.

For comparison, we mark the dynamic structures on the cavity boundaries as $\mathrm{O} 1, \mathrm{O} 2, \mathrm{O} 3$, and $\mathrm{O} 4$, and the dynamic plasma structure inside the cavity is marked as I1 (Fig. 2b). The characteristic parameters are summarized in Table 2 . The asymmetric unmagnetized plasma density enhancement (I1) of $\sim 64.5$ per cent with a duration of $\sim 188 \mathrm{~s}$ and a rising-to-descending slope ratio of $\sim 3.4$ (skewness $\sim 0.61$ ) inside the diamagnetic cavity is smaller in amplitude compared to the compressible steepened plasma structures at the contact surface. It is interesting to note that parts of the plasma structures marked as $\mathrm{O} 3$ and $\mathrm{O} 4$ are inside the cavity and parts are outside (Fig. 2b). Discontinuities can be observed in the boundaries between the magnetized and unmagnetized parts.

\subsection{Statistical results}

For the present statistical study, we identified 23 unmagnetized plasma enhancements, as shown in the above case studies, with the magnetic field magnitude $B_{o} \lesssim 1 \mathrm{nT}$ and no neutral density $n_{\text {neutral }}$ variation observed during the plasma density variation. It may be mentioned that among the 665 diamagnetic cavity crossings identified with the RPC-MAG (Goetz et al. 2016a), RPC-MIP plasma measurements were available only for $\sim 300$ cases depending on the RPC-MIP operational and telemetered data rate mode. Among these cases with RPC-MIP data $(\sim 300)$, we selected $\sim 155$ cavity crossings with durations $\geq 5 \mathrm{~min}$, long enough for reliable analysis of the internal plasma structures. About 15 per cent of them exhibited unmagnetized plasma density enhancements. These are used for the present statistical analysis. Although we explored all the cavity crossings identified by Goetz et al. (2016a), the unmagnetized plasma density enhancements are observed only during 2015 July-August and November-December. It may be mentioned that from September to the beginning of 2015 November Rosetta was far from the 67P nucleus, thus very unlikely to detect any cavity. During the detection of the unmagnetized plasma enhancements, the cometocentric distance $R$ of the Rosetta varied between $\sim 100$ and $\sim 220 \mathrm{~km}$ from the comet $67 \mathrm{P}$, whereas the estimated normalized (to the electron exobase) distance $R^{*}$ varied between $\sim 0.8$ and 1.8. We investigated whether this observation could be biased by the fact that the number of diamagnetic regions crossed by Rosetta increases with decreasing $R^{*}$ (Henri et al. 2017). For all the cavity crossings studied by Henri et al. (2017), the median value of the $R^{*}$ was estimated to be $\sim 1.85$, implying that about half of the diamagnetic regions encountered by Rosetta were observed below $R^{*}=1.85$. On the other hand, all the 23 unmagnetized plasma density enhancements were detected below $R^{*}=1.8$, whereas none of them were detected for higher $R^{*}$ values (present work). This indicates that the observation of unmagnetized plasma density enhancements close to the electron exobase is statistically significant.

The plasma characteristic parameters, namely, the duration, $\Delta N / N$ amplitude, and the asymmetry in terms of the rising-todescending slope ratio and the skewness of each of the unmagnetized plasma enhancement events, are shown in Fig. 3 and are summarized in Table 3. The histograms on the right-hand panels of Fig. 3 show that the parameters are quite well-defined (further evident from the standard deviations in Table 3). The duration varies from 76 to $278 \mathrm{~s}$ with an average duration of $\sim 2.7$ (standard deviation $(\mathrm{SD}) \pm 0.9$ ) $\mathrm{min}$. The enhancement varies from $\sim 23$ per cent to 115 per cent of the background plasma density with an average enhancement of $\sim 54$ per cent ( \pm 22 per cent), or an average $\Delta N / N$ amplitude of $\sim 0.5( \pm 0.2)$. As plasma density variation below $\sim 10$ per cent would not be detected, $\Delta N / N<10$ percent is shaded in the plot. The rising-to-descending slope ratio changes from $\sim 0.9$ to 9.7 with an average value of $\sim 2.8( \pm 1.9)$ for all of the 23 cases. Finally, the asymmetry index/skewness of the structures

Table 2. Plasma characteristics near the diamagnetic cavity crossings at $\sim 06$ UT on 2015 July 30.

\begin{tabular}{lcccc}
\hline & Duration $(\mathrm{s})$ & $\Delta N / N($ per cent) & Rising-to-descending slope ratio & Skewness \\
\hline I1 (unmagnetized) & 188 & 64.5 & 3.4 & 0.61 \\
O1 & 101 & 171.8 & 9.6 & 1.05 \\
O2 & 160 & 118.8 & 2.5 & 1.07 \\
O3 & 105 & 148.9 & 1.1 & 0.48 \\
O4 & 128 & 125.0 & 2.2 & 0.51 \\
\hline
\end{tabular}



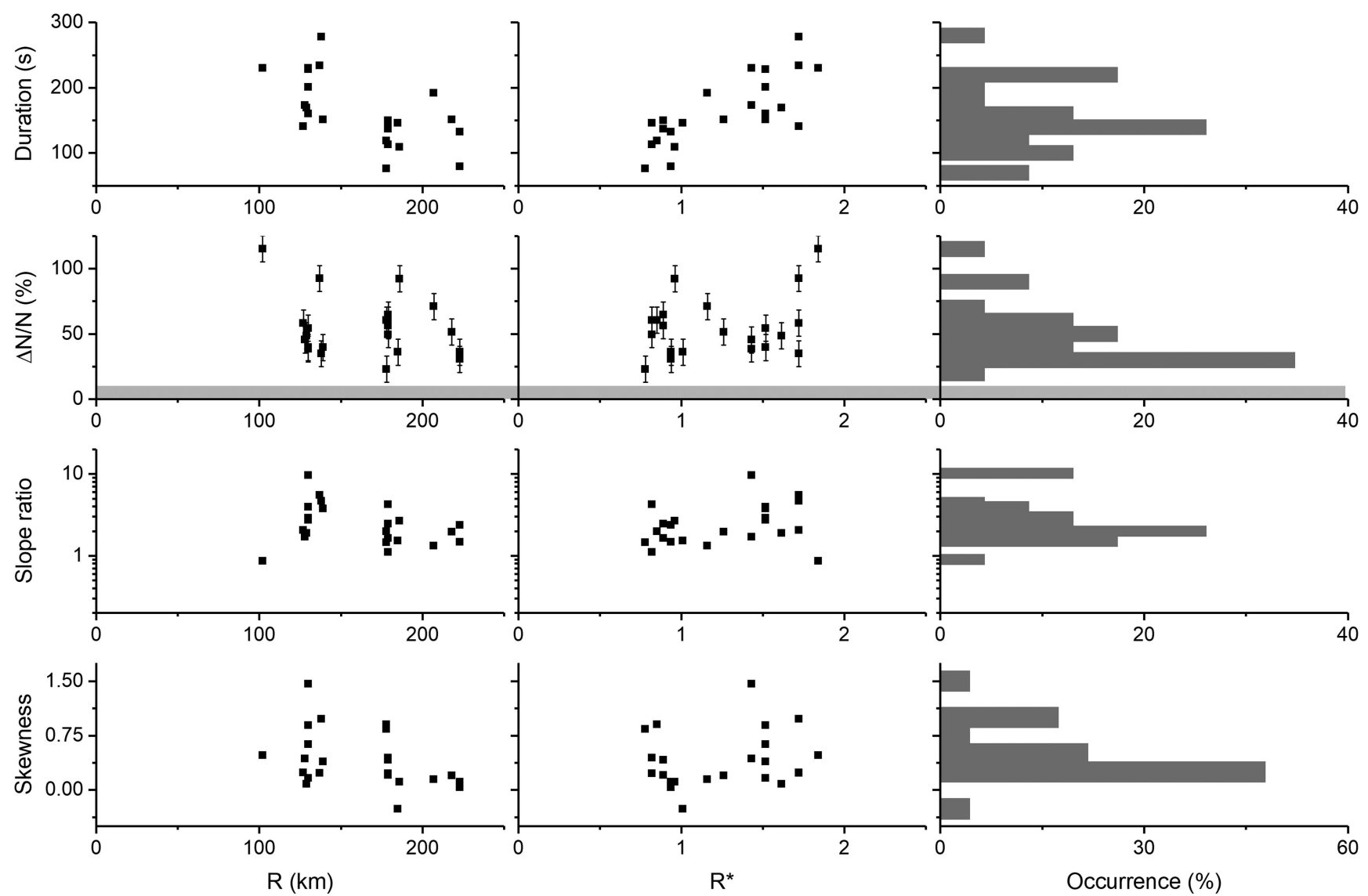

Figure 3. Variation of the duration, amplitude, and asymmetry in terms of the rising-to-descending slope ratio and skewness of the enhanced plasma structures. The left-hand and middle panels show the dependences on $R$ and $R^{*}$, respectively, whereas the right-hand panel shows the histograms. The uncertainty in $n_{\text {plasma }}$ measurement is shown by horizontal shaded region and error bars in plasma enhancement amplitude.

Table 3. Statistical characteristics of 23 unmagnetized plasma enhancements (inside diamagnetic cavity crossings).

\begin{tabular}{lcc}
\hline & Mean $( \pm \mathrm{SD})$ & Median \\
\hline Duration (s) & $163( \pm 52)$ & 151 \\
$\Delta N / N$ (per cent) & $54( \pm 22)$ & 49 \\
Rising-to-descending slope ratio & $2.8( \pm 1.9)$ & 2.1 \\
Skewness & $0.43( \pm 0.36)$ & 0.41 \\
\hline
\end{tabular}

changes from $\sim-0.26$ to 1.46 with an average skewness of $\sim 0.43$ $( \pm 0.36)$.

The left-hand and middle panels of Fig. 3 show the variations of these characteristic parameters with the cometocentric distance $R$ and the normalized cometocentric distance $R^{*}$, respectively. There are no clear dependences of the amplitude and asymmetry of the plasma enhancements on $R$ and/or $R^{*}$. However, the duration of the plasma enhancements (as encountered by the Rosetta spacecraft) increases with decreasing $R$ and increasing $R^{*}$. The duration is anticorrelated to $R$ and correlated to $R^{*}$ with correlation coefficients of $r=-0.62$ and 0.76 , respectively. The relationships are statistically significant at the 99.8 per cent and 99.9 per cent confidence levels, respectively (Student's t-test; Student 1908).

\section{DISCUSSION AND CONCLUSIONS}

We report, for the first time, the presence of sudden, large-amplitude, steepened plasma enhancements inside the diamagnetic cavities around the comet $67 \mathrm{P}$ nucleus. These were observed when the Rosetta spacecraft was located near the electron-neutral collisionopause (electron exobase). Detailed case studies were shown for the plasma enhancement events occurring around $\sim 06$ UT and $\sim 09$ UT on 2015 July 30 followed by statistical studies on 23 such plasma enhancement events inside the magnetic field-free regions. The unmagnetized plasma enhancements have asymmetric steepened structures with fast increases followed by slower decreases. The increases over the background plasma density are significantly high ( $\sim 23-115$ per cent $)$, and Rosetta encounters them for significantly long intervals of time (76-278 s). These unmagnetized plasma enhancements are found to be comparable to the magnetized steepened plasma structures, observed near the diamagnetic cavity boundaries, in terms of asymmetry and duration, though smaller in term of relative density variation.

\section{What is the nature of these steepened unmagnetized plasma enhancement structures?}

We wonder what these steepened unmagnetized plasma enhancement structures are. Are they localized coherent structures such as shocks? This could be answered by the Rankine-Hugoniot conservation analyses (Abraham-Shrauner 1972; Smith 1985; Tsurutani \& Lin 1985; Tsurutani et al. 2011; Hajra et al. 2016), for which upstream and downstream plasma velocity measurements would be required. Such measurements have been difficult to obtain because the cometary ion energy in the spacecraft frame is of the 
order of the spacecraft potential, and the time resolution of 3D ion measurements does not allow temporal resolution of the steepened compressible structures described in this paper. None the less, to identify the nature of these steepened unmagnetized plasma enhancement structures, we hereafter consider two different hypotheses: (i) that they are propagating wave-like structures, or (ii) that they are non-propagating structures and are advected in the plasma flow.

Given that the plasma is unmagnetized, we first consider these structures to propagate with the ion-acoustic velocity (hypothesis i). This consideration can be supported by the fact that Gunell et al. (2017) reported (much smaller scale) acoustic waves inside the diamagnetic cavity. The maximum possible ion-acoustic velocity of the enhanced plasma structures [dominated by water group ions, e.g. $\mathrm{H}_{2} \mathrm{O}^{+}, \mathrm{H}_{3} \mathrm{O}^{+}$, as shown by Ion Composition Analyzer (RPC-ICA; Nilsson et al. 2007) and Double Focusing Mass Spectrometer (ROSINA/DFMS; Fuselier et al. 2016) measurements] can be estimated as $C_{s}=\sqrt{ }\left(k_{\mathrm{B}} T_{\mathrm{e}} / m_{i}\right) \sim 5 \mathrm{~km} \mathrm{~s}^{-1}$, where $k_{\mathrm{B}}$ is the Boltzmann constant, $T_{\mathrm{e}}$ is the electron temperature $\left(k_{\mathrm{B}} T_{\mathrm{e}} \sim 5 \mathrm{eV}\right.$; Odelstad et al. 2015), and $m_{i}$ is the $\mathrm{H}_{2} \mathrm{O}^{+}$mass $\left(\sim 3 \times 10^{-26} \mathrm{~kg}\right)$. Here, we consider the upper boundary for the ion-acoustic velocity by considering warm electrons, while the diamagnetic region is also partially filled with cold electrons $(<0.1 \mathrm{eV}$; Eriksson et al. (2017)). In a plasma filled with cold electrons only, the ion-acoustic velocity may be as low as $C_{s} \sim 0.7 \mathrm{~km} \mathrm{~s}^{-1}$. However, strongly negative spacecraft potential measurements from the RPC-LAP instrument during diamagnetic cavity crossings suggest that a significant part of the electrons is in the $5 \mathrm{eV}$ range, which would bring the ion-acoustic velocity close to the $5 \mathrm{~km} \mathrm{~s}^{-1}$ range, mentioned above. The plasma enhancements inside the diamagnetic cavities have a mean duration of $\sim 2.7 \mathrm{~min}$. Therefore, the steepened ion-acoustic-like structures would have a scalelength between $\sim 110 \mathrm{~km}$ (for cold electrons) to a more probable $\sim 800 \mathrm{~km}$ (for warm electrons).

Now we consider standing enhanced plasma structures advected by the plasma flow (hypothesis ii). The structures therefore move with the differential speed between the Rosetta spacecraft and the plasma. As the spacecraft moves with a very slow velocity ( $<1 \mathrm{~m} \mathrm{~s}^{-1}$ in the comet frame), the differential speed is essentially the plasma velocity itself. The near-comet plasma velocity close to perihelion is estimated to be at least equal to the neutral velocity, $\sim 1 \mathrm{~km} \mathrm{~s}^{-1}$, and has been shown to decouple from the neutral flow and reach velocities up to $2-5 \mathrm{~km} \mathrm{~s}^{-1}$ (Vigren et al. 2017). In that case, the advected plasma enhancements inside the diamagnetic cavity observed during $\sim 2.7 \mathrm{~min}$ would have a scalelength between $\sim 150$ and $\sim 800 \mathrm{~km}$.

Note that both estimations (i) and (ii) are independent of the direction of propagation of the structures, which will be discussed further below. Clearly, in both cases (i) and (ii), the scalelengths of the unmagnetized plasma enhancements would be of the order of or significantly larger than the distance between the Rosetta spacecraft and the comet $67 \mathrm{P}(\sim 100-220 \mathrm{~km})$ when the steepened structures were detected, close to the edge of the diamagnetic cavity. This would mean that they would be larger than the size of the cavity itself and would fill in the entire diamagnetic cavity down to the comet nucleus. Therefore, these large-scale plasma enhancements are very unlikely to be localized plasma shocks or other local coherent structures propagating or advected in the unmagnetized cometary plasma. Instead, they most probably are global plasma enhancements that fill in a large part (if not all) of the unmagnetized plasma between the diamagnetic region boundary and the comet nucleus.

\section{What is the direction of propagation of the unmagnetized enhanced plasma density structures?}

The plasma density enhancements could initiate from the inner unmagnetized region or the outer magnetized region, and propagate, respectively, away from or towards the comet nucleus. The possibility that they are propagating from the comet nucleus can be tested by simple estimation of the typical diffusion time of the plasma structures in the near-nucleus region, where the plasma dynamics are expected to be dominated by ion-neutral collisions. For $n_{\text {neutral }}$ $\sim 6 \times 10^{7} \mathrm{~cm}^{-3}$ measured by the Rosetta at cometocentric distance $R \sim 100-200 \mathrm{~km}$, the location of the ion-neutral collisionopause can be estimated as $L_{\text {in }}=\sigma_{\text {in }} n_{\text {neutral }} R^{2} \sim 480-2300 \mathrm{~km}$ (Mandt et al. 2016). The ion-neutral cross-section $\sigma_{\text {in }}$ is considered to be $\sim 8 \times 10^{-15} \mathrm{~cm}^{2}$ (Mendis et al. 1986). Thus, Rosetta was well inside the ion-neutral collisional region during the period of observations. As the plasma structure is composed of water group ions, the diffusion coefficient (diffusivity) of the plasma can be estimated as $D_{i} \sim\left\langle v_{i}\right\rangle^{2} / v_{\text {in }}$, where $\left\langle v_{i}\right\rangle$ is the mean ion velocity, and $v_{\text {in }}$ is the collision frequency for scattering of $\mathrm{H}_{2} \mathrm{O}^{+}$ions by neutrals. The $v_{\text {in }}$ is given by $n_{\text {neutral }} \sigma_{\text {in }}\left\langle v_{\text {in }}^{r}\right\rangle$, where $\left\langle v_{\text {in }}^{r}\right\rangle$ is the mean relative velocity between the ions and neutrals. The diffusion time-scale $t_{\mathrm{D}}$ depends on the mean free path $\lambda_{f}$ and the diffusivity $D_{i}$ as $t_{\mathrm{D}}=\lambda_{f}^{2} / 2 D_{i}$ (Einstein-Smoluchowski equation), where the $\mathrm{H}_{2} \mathrm{O}^{+}$mean free path can be estimated as $\lambda_{f}=\left(n_{\text {neutral }} \sigma_{\text {in }}\right)^{-1}$. Thus, the diffusion time-scale can be expressed as follows:

$t_{\mathrm{D}} \sim \frac{1}{2} \frac{\left\langle v_{\text {in }}^{r}\right\rangle}{n_{\text {neutral }} \sigma_{\text {in }}\left\langle v_{i}\right\rangle^{2}}$.

The neutral density $n_{\text {neutral }}$ has been shown to follow an $R^{-2}$ dependence (Hässig et al. 2015; Bieler et al. 2015). If the neutral velocity is taken to be $\sim 1 \mathrm{~km} \mathrm{~s}^{-1}$ and the ion velocity $\left\langle v_{i}\right\rangle$ as $\sim 3 \mathrm{~km} \mathrm{~s}^{-1}$ (see fig. 6 of Vigren et al. 2017 for ion velocity during plasma enhancement inside a diamagnetic cavity), $\left\langle v_{\text {in }}^{r}\right\rangle$ varies between $\sim 2$ and $4 \mathrm{~km} \mathrm{~s}^{-1}$. In the present scenario, the expected typical diffusion time $t_{\mathrm{D}}$ of the plasma enhancement structures varies with the cometocentric distance $R$ as $t_{\mathrm{D}} \sim 0.7-1.4 \times 10^{-4} R^{2}$, where $R$ is given in $\mathrm{km}$ and $t_{\mathrm{D}}$ is expressed in s. At cometocentric distance of $\sim 100-220 \mathrm{~km}$, where the Rosetta detected the plasma enhancements, the structures are expected to diffuse in $\sim 1-7 \mathrm{~s}$. Compared to these time-scales, the recombination time-scale $\left(\sim 10^{6} \mathrm{~s}\right)$ of the water ions with electrons is much larger, which cannot explain the descending slope of the structures. This process can be neglected for the present case. However, the diffusion time is expected to be much faster closer to the $67 \mathrm{P}$ nucleus. For example, at $\sim 10 \mathrm{~km}$ from the $67 \mathrm{P}$, they will diffuse one hundred times faster, on time-scales much smaller than the ballistic time-scale required to reach the cometocentric distance where they are observed. Therefore, if some overdense plasma structures initiated from the inner coma close to the nucleus, they could hardly be observed by Rosetta. In addition, there is, to our knowledge, no known mechanism to create large plasma enhancements close to the nucleus with no corresponding neutral gas enhancement. Such a local neutral enhancement close to the nucleus would also diffuse and should be seen correlated with the plasma enhancement at the observation point. Thus, the long-duration (76-278 s), large-amplitude, asymmetric plasma structures are unlikely to be propagated from the comet outward as they would have been diffused owing to collisions with neutrals before they could be detected by the Rosetta spacecraft instrumentation. In other words, the preferred hypothesis is that the unmagnetized plasma enhancements propagate towards the comet. This is consistent with a significant motion of cometary ions in the antisunward direction observed for 


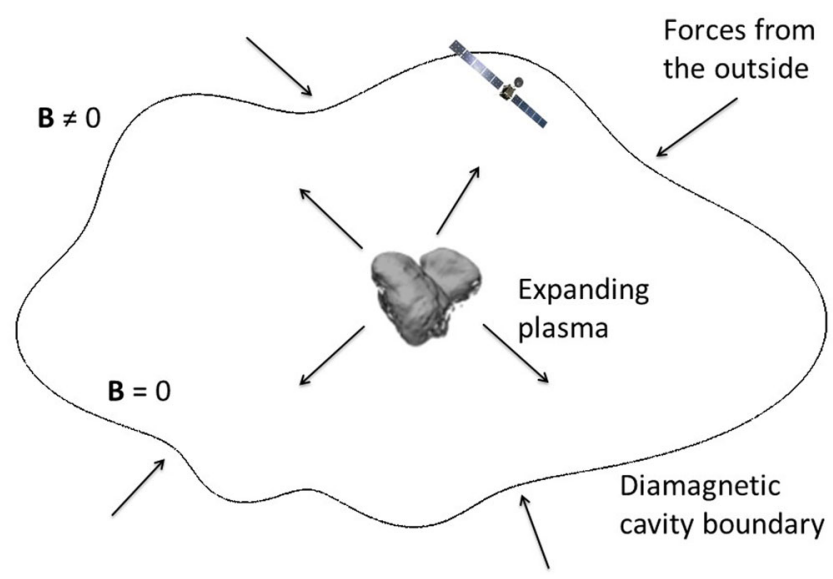

Figure 4. Schematic of diamagnetic cavity around the comet 67P.

this period (Nilsson et al. 2017), whereas the detailed ion flow can be more complicated and must await another study based on ion spectrometer data. At the same time, we observed identical steepened compressible/dynamic plasma structures outside the diamagnetic cavities. Thus, we may conclude that the outside magnetized steepened compressible plasma structures are transmitted inside the diamagnetic cavities. It may be recalled that the Rosetta orbiter encountered the enhanced plasma structures for longer duration near the comet compared to those far from the nucleus. This result is consistent with fast diffusion, leading to larger scattering through ion-neutral collisions when moving closer to the comet nucleus. The scenario described in the above discussion is schematically shown in Fig. 4. The Rosetta spacecraft is shown to pass through the unstable cavity boundary under forces from outside the cavity. As indicated, the density pulses most likely come from the cavity boundary. However, this does not mean that they move radially inwards or come from the part of the boundary that is closest to the spacecraft, as the boundary can be hit from the outside in many different places.

\section{Transmission mechanism across the diamagnetic cavity boundary}

The second example described in Section 2.2 (Fig. 2), where signatures of both an unmagnetized and a magnetized plasma density enhancements are observed on both sides of a diamagnetic region boundary, is thus likely to show a magnetized steepened compressible plasma structure being transmitted inside the diamagnetic cavity. The nature and origin of such magnetized steepened structures, still to be understood, will be addressed in future works. Interestingly, the observations show that the relative density increase $\Delta N / N$ is larger for these magnetized steepened structures than for the unmagnetized ones observed in the diamagnetic region. This should give clues on the plausible transmission mechanism.

If the unmagnetized enhanced density structures are transmitted from the outer magnetized region, it is still unclear whether the excess plasma comes from the outer region (mass transport across a permeable diamagnetic cavity boundary) or from the inner region (global compression of an impermeable diamagnetic cavity boundary). Although the outer cometary plasma is essentially composed of the warm electron population, the inner cometary plasma has been shown to be composed of a mixture of warm $(\sim 5 \mathrm{eV})$ and cold $(<1 \mathrm{eV})$ electrons, the latter being thermalized by collisions with the cold cometary neutrals (Eriksson et al. 2007). Based on the method described in Gilet et al. (2017), we performed preliminary studies to separate the relative density variations of these two electron populations for the enhanced unmagnetized plasma structures observed during 2015 November (not shown). It is suggested that the cometary plasma, both inside and outside the diamagnetic cavity, was a mixture of cold and warm electrons with a cold-to-total density ratio of $\sim 70$ per cent. Although the cold electron population is estimated to be dominating, the relative contribution of the cold and warm electron populations seems to remain unchanged throughout the cavity and during the unmagnetized plasma enhancements as well. This may help constraining the level of mass transport during the transmission of magnetized to unmagnetized plasma steepened structures, and therefore gives clues on the level of permeability of the diamagnetic cavity boundary. This issue should be addressed in more details in future works.

Finally, it should be mentioned that the estimations of the scalelength and propagation direction of the structures are difficult because of single spacecraft (Rosetta) measurements. It is still not clear why only few of the high plasma density regions detected outside the diamagnetic regions are observed inside. Further modelling may be explored to better understand this unexpected cometary plasma phenomenon.

\section{ACKNOWLEDGEMENTS}

Rosetta is the European Space Agency (ESA) mission with contributions from its member states and National Aeronautics and Space Administration (NASA). The work at LPC2E/CNRS was supported by CNES, ESEP, and by ANR under the financial agreement ANR15-CE31-0009-01. The work on RPC-MAG was financially supported by the German Ministerium für Wirtschaft und Energie and the Deutsches Zentrum für Luft- und Raumfahrt under contract 50QP 1401. Work on ROSINA/COPS at the University of Bern was funded by the State of Bern, the Swiss National Science Foundation, and by the ESA PRODEX program. Work at the Royal Belgian Institute for Space Aeronomy was supported by the Belgian Science Policy Office through the Solar Terrestrial Centre of Excellence and by PRODEX/ROSETTA/ROSINA PEA 4000107705. The work of ZN was supported by the János Bolyai Research Scholarship of the Hungarian Academy of Sciences. Portions of the research were conducted at the Jet Propulsion Laboratory, California Institute of Technology, under contract with NASA. This work has made use of AMDA and RPC quick-look data base to provide an initial overview of the event studied. This is provided through a collaboration between the Centre de Données de la Physique des Plasmas (CDPP) (supported by CNRS, CNES, Observatoire de Paris, and Université Paul-Sabatier, Toulouse) and Imperial College London (supported by the UK Science and Technology Facilities Council). The data used in this paper will soon be made available on the ESA planetary science archive and are available upon request until that time.

\section{REFERENCES}

Abraham-Shrauner B., 1972, J. Geophys. Res., 77, 736

Balsiger H. et al., 2007, Space Sci. Rev., 128, 745

Behar E., Nilsson H., Alho M., Goetz C., Tsurutani B., 2017, MNRAS, 469, S396

Bieler A. et al., 2015, A\&A, 583, A7

Churyumov K. I., Gerasimenko S. I., 1972, in Chebotarev G. A., Kazimirchak-Polonskaya E. I., Marsden B. G., eds, IAU Symp. 45, The Motion, Evolution of Orbits, and Origin of Comets. Springer Netherlands, Dordrecht, p. 27 
Cravens T. E., 1987, Adv. Space Res., 7, 147

Eriksson A. I. et al., 2007, Space Sci. Rev., 128, 729

Eriksson A. I. et al., 2017, A\&A, 605, A15

Fuselier S. A. et al., 2016, MNRAS, 462, S67

Galand M. et al., 2016, MNRAS, 462, S331

Gilet N., Henri P., Wattieaux G., Cilibrasi M., Béghin C., 2017, Radio Science, 52, 1432

Glassmeier K.-H., Boehnhardt H., Koschny D., Kührt E., Richter I., 2007a, Space Sci. Rev., 128, 1

Glassmeier K. -H. et al., 2007b, Space Sci. Rev., 128, 649

Goetz C. et al., 2016a, MNRAS, 462, S459

Goetz C. et al., 2016b, A\&A, 588, A24

Gunell H. et al., 2017, MNRAS, 469, S84

Hajra R., Tsurutani B. T., Echer E., Gonzalez W. D., Gjerloev J. W., 2016, J. Geophys. Res., 121, 7805

Hajra R. et al., 2017, A\&A, 607, A34

Hässig M. et al., 2015, Science, 347, aaa0276

Henri P. et al., 2017, MNRAS, 469, S372

Heritier K. L. et al., 2017a, MNRAS, 469, S118

Heritier K. L. et al., 2017b, MNRAS, 469, S427

Ip W.-H., Axford W. I., 1987, Nature, 325, 418
Itikawa Y., Mason N., 2005, J. Phys. Chem. Ref. Data, 34, 1

Madanian H. et al., 2017, Astron. J., 153, 30

Mandt K. E. et al., 2016, MNRAS, 462, S9

Mendis D. A., Smith E. J., Tsurutani B. T., Slavin J. A., Jones D. E., Siscoe

G. L., 1986, Geophys. Res. Lett., 13, 239

Nemeth Z. et al., 2016, MNRAS, 462, S415

Neubauer F. M. et al., 1986, Nature, 321, 352

Nilsson H. et al., 2007, Space Sci. Rev., 128, 671

Nilsson H. et al., 2017, MNRAS, 469, S252

Odelstad E. et al., 2015, Geophys. Res. Lett., 42, 10126

Smith E. J., 1985, AGU, Washington, DC

Student, 1908, Biometrika, 6, 1

Trotignon J. G. et al., 2007, Space Sci. Rev., 128, 713

Tsurutani B. T., Lin R. P., 1985, J. Geophys. Res., 90, 1

Tsurutani B. T., Lakhina G. S., Verkhoglyadova O. P., Gonzalez W. D., Echer E., Guarnieri F. L., 2011, J. Atmos. Sol.-Terr. Phys., 73, 5

Vigren E. et al., 2017, MNRAS, 469, S142

This paper has been typeset from a $\mathrm{T}_{\mathrm{E}} \mathrm{X} / \mathrm{LT} \mathrm{E} \mathrm{X}$ file prepared by the author. 